\title{
CONTRIBUIÇÕES DA ENFERMAGEM NA REESTRUTURAÇÃO DO SERVIÇO DE CIRURGIA DE JOELHO DE UM AMBULATÓRIO ESCOLA
}

\section{CONTRIBUTIONS OF NURSING TO RESTRUCTURE THE KNEE SURGERY SERVICE OF A SCHOOL AMBULATORY}

\section{CONTRIBUCIONES DE LA ENFERMERÍA A LA REESTRUCTURACIÓN DEL SERVICIO DE CIRUGÍA DE RODILLA EN UN HOSPITAL ESCUELA}

\author{
Déborah Priscilla Oliveira Almeida ${ }^{1}$, Carla Aparecida Spagnol ${ }^{2}$, Amália Augusta Nunes $^{3}$, \\ Letícia Gonçalves Figueiredo ${ }^{4}$, Caroliny Alves Pessoa ${ }^{5}$, Isabela Silva Cancio Velloso ${ }^{6}$, Vania \\ Regina Goveia ${ }^{7}$, Biannka Melo dos Santos ${ }^{8}$, Mhayara Cardoso dos Santos ${ }^{9}$
}

Como citar esse artigo: Almeida DPO, Spagnol CA, Nunes AA, Figueiredo LG, Pessoa CA, Velloso ISC, Goveia VR, Santos BM, Santos MC. Contribuições da enfermagem na reestruturação do serviço de cirurgia de joelho de um ambulatório escola. Rev Enferm Atenção Saúde [Internet]. 2021 [acesso em ];10(2):e202120. doi:10.18554/reas.v10i2.5034

\begin{abstract}
RESUMO
Objetivo: relatar a experiência de reestruturação do serviço de cirurgia de joelho, de um ambulatório escola, tendo em vista a sistematização da assistência de enfermagem prestada aos usuários que serão submetidos à artroplastia de joelho. Método: relato de experiência das ações do planejamento estratégico realizado, no período de 2016 a 2018, em um ambulatório localizado em Belo Horizonte. Resultados: Os principais resultados foram: elaboração coletiva de uma linha de cuidado; implementação de um grupo educativo multidisciplinar e elaboração de materiais didáticos. As estratégias de educação em saúde utilizadas fornecem informações pertinentes à segurança e recuperação do paciente no período perioperatório de cirurgia de joelho, estimulando o autocuidado. Conclusão: a continuidade deste trabalho é de fundamental importância para a consolidação destas conquistas, além da realização de outros
\end{abstract}

1 Enfermeira. Especializanda no Hospital Metropolitano Odilon Behrens, Urgência e Emergência. Belo Horizonte, Minas Gerais - Brasil.

2 Enfermeira. Professora Associada da Escola de Enfermagem do Departamento de Enfermagem Aplicada da Universidade Federal de Minas Gerais, nos cursos de graduação em enfermagem e Mestrado Profissional em Gestão de Serviços de Saúde. Belo Horizonte, Minas Gerais - Brasil.

3 Enfermeira do Ambulatório Bias Fortes do Hospital das Clínicas da Universidade Federal de Minas Gerais. Belo Horizonte, Minas Gerais - Brasil.

4 Enfermeira do Ambulatório Bias Fortes do Hospital das Clínicas da Universidade Federal de Minas Gerais. Belo Horizonte, Minas Gerais - Brasil.

5 Enfermeira do Ambulatório Bias Fortes do Hospital das Clínicas da Universidade Federal de Minas Gerais. Belo Horizonte, Minas Gerais - Brasil.

6 Enfermeira. Professora Associada da Escola de Enfermagem do Departamento de Enfermagem Aplicada da Universidade Federal de Minas Gerais. Belo Horizonte, Minas Gerais - Brasil.

7 Enfermeira. Professora Associada da Escola de Enfermagem do Departamento de Enfermagem Básica da Universidade Federal de Minas Gerais. Belo Horizonte, Minas Gerais - Brasil.

8 Aluna do curso de graduação em enfermagem da Escola de Enfermagem da Universidade Federal de Minas Gerais, bolsista voluntária da iniciação à extensão. Belo Horizonte, Minas Gerais - Brasil.

9 Aluna do curso de graduação em enfermagem da Escola de Enfermagem da Universidade Federal de Minas Gerais, bolsista voluntária da iniciação à extensão. Belo Horizonte, Minas Gerais - Brasil. 
estudos e pesquisas, a fim de contribuir para o avanço do conhecimento na área da saúde e na enfermagem ortopédica no ambulatório de especialidades.

Descritores: Planejamento Estratégico; Enfermagem Perioperatória; Ortopedia; Instituições de Assistência Ambulatorial; Educação em Saúde.

\begin{abstract}
Objective: to report the experience of restructuring the knee surgery service in a school clinic, with a view to systematizing the nursing care delivered to people who will be submitted to total Knee replacement. Method: experience report of the strategic planning actions carried out from 2016 to 2018, in an outpatient clinic in Belo Horizonte. Result: The main results were: collective elaboration of a line of care; implementation of a multidisciplinary educational group and courseware development. Health education strategies provided relevant information about patient safety and recovery in knee surgery perioperative period, encouraging self-care. Conclusion: the continuity of this work is of fundamental importance for the consolidation of these achievements, in addition to other studies and research, in order to contribute to the advancement of knowledge in the area of health and in orthopedic nursing in outpatient clinic. Descriptors: Strategic Planning; Perioperative Nursing; Orthopedics; Ambulatory Care Facilities; Health Education.
\end{abstract}

\title{
RESUMEN
}

Objetivo: relatar la experiencia de reestructuración del servicio de cirugía de rodilla de un hospital escuela, a fin de sistematizar la atención de enfermería brindada a los usuarios que serán sometidos a artroplastia total de rodilla. Metodología: relato de experiencia sobre las acciones de planificación estratégica realizadas de 2016 a 2018. Resultados: los principales resultados fueron: elaboración colectiva de esquema de atención, implementación de un grupo educativo multidisciplinario y elaboración de material didáctico. Las estrategias de educación para la salud utilizadas brindan información relevante para la seguridad y recuperación del paciente durante el período perioperatorio de cirugía de rodilla, estimulando el autocuidado. Conclusión: la continuidad de este trabajo es fundamental para consolidar estos logros y para realizar otros estudios e investigaciones, a fin de contribuir al avance del conocimiento en el área de la salud y de la enfermería ortopédica en el servicio ambulatorio de especialidades.

Descriptores: Planificación Estratégica; Enfermería Perioperatoria; Ortopedia; Instituciones de Atención Ambulatoria; Educación para la Salud.

\section{INTRODUÇÃOO}

O planejamento estratégico situacional (PES) é uma ferramenta de gestão direcionada para a resolução de problemas em que os atores sociais efetivamente participam do seu processo. ${ }^{1}$ Assim, o PES foi elaborado em um ambulatório de especialidades, tendo em vista a reorganização do processo de trabalho e a melhor qualificação da assistência prestada.

No processo de planejamento das ações de enfermagem priorizou-se a sistematização do atendimento aos pacientes que seriam submetidos a cirurgia de joelho, dentre elas a artroplastia total de joelho (ATJ), devido à sua especificidade, complexidade e o 
número cirurgias realizadas no mês (no mínimo 20).

A ATJ é uma das indicações para o tratamento de osteoartrite (OA), sendo o procedimento mais realizado e de maior sucesso, tornando o joelho mais funcional e criando uma reconstrução durável. A OA é uma disfunção articular, com caráter inflamatório e degenerativo, resultante de eventos biológicos e mecânicos, sendo sua prevalência em pessoas acima de 60 anos. ${ }^{2,3}$ Portanto, considerando o envelhecimento populacional, espera-se grande aumento do número de casos de $\mathrm{OA} e$, consequentemente, aumento nas cirurgias, sobretudo no Brasil, onde $4 \%$ da população é acometida por essa patologia. ${ }^{3}$

$\mathrm{Na}$ ATJ todas as superfícies articulares são substituídas por peças metálicas ou de polietileno. O objetivo principal da cirurgia é garantir o alinhamento do joelho, alívio da dor e obter estabilidade. É um procedimento considerado de grande porte e de alto custo, que necessita de recursos médicos e hospitalares adequados para sua realização. Devido a isto, quanto mais familiarizados o paciente e a equipe estiverem com o processo da intervenção cirúrgica, melhores serão os resultados. ${ }^{2}$

Sendo assim, a educação em saúde pode proporcionar resultados positivos, constituindo-se uma estratégia fundamental para que o paciente receba as informações adequadas no período que compreende o perioperatório. ${ }^{4}$ Um estudo realizado com pacientes submetidos à cirurgia cardíaca revelou que o fato de os mesmos poderem expressar no grupo educativo seus sentimentos e expectativas exerceu influência positiva na redução da ansiedade. $^{5}$

$$
\mathrm{O} \text { ponto de partida para }
$$
sistematizar o atendimento no serviço de cirurgia de ATJ foi a elaboração da linha de cuidado da "Reconstrução Articular", que preconizou uma assistência multiprofissional e interdisciplinar, que tivesse interface com ações de educação em saúde.

Nesta direção, o serviço de ortopedia do ambulatório preconizou em sua linha de cuidado, o grupo educativo no pré-operatório, como uma das estratégias de educação em saúde, que tem a finalidade de orientar os pacientes quanto aos cuidados nas cirurgias de ATJ, com vistas a reduzir a ansiedade e o medo do desconhecido presente em todas as fases do processo cirúrgico.

O presente trabalho tem como objetivo relatar a experiência de reestruturação do serviço de cirurgia de joelho, de um ambulatório escola, tendo em vista a sistematização da assistência de enfermagem prestada aos usuários que serão submetidos à artroplastia de joelho. 


\section{MÉTODO}

Trata-se de um relato de experiência, descritivo e analítico, das ações desenvolvidas, no período de 2016 a 2018, na reestruturação do serviço de cirurgia de joelho de um ambulatório, vinculado à um hospital universitário público de Belo Horizonte, Minas Gerais.

Esse trabalho faz parte de um Projeto de Extensão que foi aprovado pelo gerente da Unidade de Atenção Ambulatorial, o que dispensou a aprovação do Comitê de Ética em Pesquisa, pois não se caracteriza como uma pesquisa, mas, sim de uma análise de situações que emergiram da prática profissional e acadêmica. No entanto, procurou-se preservar a identidade das instituições em análise e dos profissionais envolvidos nas atividades.

$\mathrm{Na}$ reestruturação do serviço foram executadas as seguintes etapas do planejamento estratégico: diagnóstico situacional (momento explicativo), plano de ação (momento normativo e estratégico) e implementação das ações (momento táticooperacional). ${ }^{1}$

Dentre os problemas elencados no diagnóstico situacional estavam a ausência da delimitação do papel do enfermeiro na ortopedia e a fragmentação da assistência prestada aos pacientes, a qual se restringia à consulta médica. A segunda etapa consistiuse na elaboração do plano de ação realizada de forma coletiva e a terceira etapa na implementação da linha de cuidado e de um grupo educativo.

\section{RESULTADOS}

A elaboração da linha de cuidado para pacientes candidatos as cirurgias de joelho, em especial à ATJ, foi a primeira intervenção do plano de ação realizada e que, aos poucos, vem construindo a transposição do modelo biomédico para o modelo de assistência usuário centrado no ambulatório de especialidades em estudo. Para isso, foram realizadas reuniões com os diretores do hospital, do qual o ambulatório faz parte, e as principais lideranças do setor, para apresentar a proposta e os pontos principais acerca da infraestrutura, dos recursos humanos e operacionais necessários à estruturação para esse tipo atendimento.

Um dos principais propósitos desta linha de cuidados é proporcionar aos pacientes com osteoartrite acesso às informações e à educação em saúde, quanto aos objetivos do tratamento e à importância de mudanças no estilo de vida, para diminuir o impacto sobre as articulações lesadas e os cuidados no pós-operatório. Isso foi feito por meio da criação de um grupo educativo multiprofissional, dentre outras ações. 
Para definição da temática a ser abordada no grupo e para elaboração de materiais educativos, foi realizada uma busca na literatura científica. Além disso, a experiência das equipes no atendimento aos pacientes ortopédicos também subsidiou a definição dos temas das orientações fornecidas no grupo educativo. Após a definição dos temas, discutiu-se com a equipe as estratégias para confecção de um material didático, optando-se, inicialmente, pela elaboração de um material multimídia, composto por slides e vídeo que facilitassem a demonstração visual dos procedimentos, equipamentos e materiais necessários ao entendimento do paciente sobre o processo da cirurgia de joelho.

O grupo educativo teve início no segundo semestre de 2016, participando em média dez pessoas, entre pacientes candidatos à cirurgia de joelho e seus acompanhantes, que compareceram ao serviço para realizar a primeira consulta. A ação educativa era realizada uma vez na semana, com duração aproximada de 60 minutos, sendo conduzida pela enfermeira do setor, alunas de enfermagem voluntárias, fisioterapeuta e médico ortopedista.

Durante a exposição dialogada dos temas, os pacientes e acompanhantes elaboravam perguntas e as dúvidas eram esclarecidas pelos profissionais e pelas acadêmicas de enfermagem. Houve uma efetiva participação dos usuários que, além Rev Enferm Atenção Saúde [Online]. Jul/Set 2021; 10(2):e202120 de dúvidas, também traziam depoimentos sobre suas experiências pessoais. Ao final, os participantes eram encaminhados à sala de espera, onde aguardavam pela consulta médica. Ao longo de dois anos de sua implantação (2016-2018), o grupo educativo contou com a participação de aproximadamente 597 pessoas, entre pacientes e acompanhantes.

A equipe ainda optou por elaborar uma cartilha contendo, de forma sucinta, as informações abordadas no grupo educativo. Esse material didático tinha o propósito de fornecer informações pertinentes à segurança e recuperação do paciente no período perioperatório de cirurgia de joelho, estimulando o autocuidado e uma melhor percepção sobre seu estado de saúde.

Em síntese, as principais conquistas do processo de reorganização do serviço de cirurgia de joelho, os produtos e resultados obtidos até então, que contaram com as contribuições da enfermagem foram: elaboração coletiva da linha de cuidado; implementação do grupo educativo multiprofissional no préoperatório e elaboração de materiais didáticos (slides e cartilha) para apresentação e distribuição aos pacientes e acompanhantes. 


\section{DISCUSSÃO}

A elaboração de uma linha de cuidado é uma ação fundamental para promover a articulação das ações e dos saberes dos membros da equipe multiprofissional, em busca da superação de uma assistência fragmentada. ${ }^{6}$ No caso da linha de cuidado da "Reconstrução articular", foi introduzido, paulatinamente, a integralidade das ações de saúde aos usuários na ortopedia, contemplando a educação em saúde.

A orientação no pré-operatório pode ser definida como qualquer atividade educativa realizada antes da intervenção cirúrgica que tenha por objetivo contribuir para o aumento do conhecimento dos envolvidos, melhora dos resultados e modificação dos comportamentos relacionados à saúde ${ }^{5}$. Uma revisão sistemática da literatura apontou que este tipo de educação em saúde geralmente é realizado por uma equipe multiprofissional e os conteúdos das suas ações, para pacientes ortopédicos, incluem os ambientes estressantes relacionados à cirurgia, as etapas do procedimento cirúrgico, os cuidados pré e pósoperatórios, possíveis complicações, controle da dor e exercícios fisioterápicos. ${ }^{7}$

Nesse sentido, ressaltam-se as evidências presentes na literatura, sobretudo no cenário internacional, as quais demonstram que as ações educativas para pacientes candidatos a ATJ, contribuem para melhora do conhecimento sobre a doença, do controle da dor, adesão e desempenho dos exercícios, além da diminuição do tempo de internação, redução dos custos e da taxa de revisão cirúrgica. $^{4}$

Os grupos educativos que fornecem o espaço e o tempo necessário para discussão e questionamentos dos pacientes e acompanhantes são benéficos à medida que permitem ao participante compartilhar respostas e perguntas de outros integrantes do grupo. Segundo os autores, os temas do grupo educativo de pré-operatório, devem ser ministrados por todos os membros da equipe multiprofissional envolvidos no seu cuidado, além de integrar a rede de apoio do paciente, e utilizar de métodos visuais como slides e vídeos em associação com a comunicação verbal. $^{8}$

A construção de material didático é uma parte essencial das intervenções educativas na saúde. Trata-se de uma tecnologia leve e leve dura que quando bem estruturada permite orientar o processo de educação, conscientizar, transformar e possibilitar a construção de vínculos de confiança entre o profissional e os usuários. ${ }^{9}$

Neste sentido, torna-se fundamental inserir atividades de educação em saúde na formação dos futuros profissionais saúde, 
pois, o seu conjunto se constituem como uma prática social que tem a finalidade de promover a reflexão e a consciência crítica dos indivíduos sobre sua situação de vida e de saúde. ${ }^{10}$

Portanto, a implementação de algumas ações, dentre elas o grupo educativo está contribuindo cada vez mais para a redefinição do papel do enfermeiro e, consequentemente, para a atuação da enfermagem no setor de ortopedia do ambulatório escola, o que proporciona a qualificação da assistência prestada, pautada no princípio da integralidade e da autonomia dos usuários e trabalhadores.

\section{CONCLUSÕES}

A partir do planejamento estratégico, foi possível dar início à reestruturação do serviço de cirurgia ortopédica do ambulatório escola. Assim, a participação dos trabalhadores da enfermagem em todas as suas etapas, desde o diagnóstico até a execução das ações foi imperativo para o sucesso alcançado.

Este comprometimento, somado ao trabalho organizado em um plano e metas, trouxe notória realização e motivação aos profissionais da enfermagem envolvidos. Esta motivação se deu pela construção e clara definição da importância e do papel da enfermagem dentro do setor e na equipe multiprofissional.

A reorganização do serviço possibilitou algumas mudanças que influenciaram a assistência no pré e pósoperatório nas cirurgias de joelho, permitindo uma participação mais efetiva do enfermeiro nos cuidados prestados aos pacientes, em especial aqueles submetidos ATJ.

A participação da equipe de enfermagem na construção da linha de cuidado "Reconstrução articular" foi uma das ações relevantes, pois foi possível constatar o seu reconhecimento junto à equipe multiprofissional. Além disso, a criação do grupo educativo é um primeiro passo para a integralidade da assistência prestada aos usuários na ortopedia e confere mais um sentido ao trabalho do enfermeiro no ambulatório.

A estruturação do grupo e a criação do material educativo constituíram-se em ações importantes para se prevenir complicações pós-operatórias, reduzir a ansiedade relacionada à intervenção cirúrgica e consequentemente melhorar da qualidade de vida dos pacientes.

A maior participação do enfermeiro facilitou a identificação de problemas, cooperando assim para integração de outros profissionais nesse cuidado, à medida que houve a integração da fisioterapia e aumento no 
encaminhamento dos pacientes para a assistente social e para a psicóloga do ambulatório. Espera-se que, com isto, a assistência à saúde no serviço seja mais efetiva e eficaz e que os resultados cirúrgicos sejam aprimorados.

Ressalta-se que essa experiência se desdobrou em um projeto de pesquisa que pretende avaliar a compreensão dos pacientes acerca das orientações realizadas e das ações adotadas frente a elas, considerando as estratégias de educação em saúde utilizadas nas cirurgias de joelho, como o grupo educativo e a cartilha.

Por fim, aponta-se a necessidade de se realizar outros estudos e pesquisas que possam contribuir para o avanço do conhecimento na área da saúde e na enfermagem ortopédica, em especial no ambulatório de especialidade.

\section{REFERÊNCIAS}

1. Silva AK, Sousa JP, Rodrigues W, Cançado AC. Planejamento estratégico situacional- PES: uma análise bibliométrica da produção científica brasileira. Rev Serv Público. [Internet]. 2017 [citado em 08 out 2019]; 68(2):365-88.

Disponível em: https://revista.enap.gov.br/index.ph p/RSP/article/view/1269. doi: https://doi.org/10.21874/rsp.v68i2. 1269

2. Barros Filho TEP, Camargo OPD, Camanho GL. Clínica Ortopédica. Barueri: Manole; 2012.
3. Ferreira MC, Oliveira JCP, Zidan FF, Franciozi CES, Luzo MVM, Abdalla RJ. Artroplastia total de joelho e quadril: a preocupante realidade assistencial do Sistema Único de Saúde brasileiro. Rev Bras Ortop. [Internet]. 2018 [citado em 07 nov 2019]; 53(4):432-40. Disponível em: https://rbo.org.br/detalhes/2711/ptBR/artroplastia-total-de-joelho-equadril--a-preocupante-realidadeassistencial-do-sistema-unico-desaude-brasileiro. doi: doi.org/10.1016/j.rboe.2018.05.002

4. Majid N, Lee S, Plummer V. The effectiveness of orthopedic patient education in improving patient outcomes: a systematic review protocol. JBI Database of System Rev Implement Rep. [Internet]. 2015 [citado em 20 ago 2019]; 12(1):122-33. Disponível em: https://pubmed.ncbi.nlm.nih.gov/26 447013/. doi: 10.11124/jbisrir2015-1950

5. Almeida SM, Souza EN, Azzolin KO. Efeito da orientação préoperatória por grupo multiprofissional na ansiedade de pacientes submetidos à cirurgia cardíaca. Rev Enferm UFSM. [Internet]. 2013 [citado em 24 jul 2019]; 3(3):402-8. Disponível em: https://periodicos.ufsm.br/reufsm/ar ticle/view/8809. doi: doi.org/10.5902/217976928809

6. Torres ARA, Barreto ICHC, Albuquerque IMAN, Gomes VB. Construção participativa de uma linha de cuidado ao trabalhador com lesão por esforços repetitivos. Rev Rene. [Internet]. 2016 [citado em 07 out 2020]; 17(5): 626-35. Disponível em: http://periodicos.ufc.br/rene/article/ view/6187. doi: /10.15253/21756783.2016000500007

7. McDonald S, Page MJ, Beringer K, Wasiak J, Sprowson A. 
Preoperative education for hip or knee replacement. Cochrane

Database Syst Rev. [Internet]. 2014

[citado em 09 nov 2019]; 1(5).

Disponível em:

https://pubmed.ncbi.nlm.nih.gov/24

820247/. doi:

10.1002/14651858.CD003526.pub3

8. Edwards PK, Mears SC, Barnes

CL. Preoperative education for hip and knee replacement: never stop learning. Curr Rev Musculoskelet Med. [Internet]. 2018 [citado em 10 set 2019]; 10(3):356-34. doi:

Disponível em:

https://pubmed.ncbi.nlm.nih.gov/28 647838/. 10.1007/s12178-0179417-4

9. Paula FMS, Beserra NCN, Lopes RCS, Guerra DR. Elaboração de material didático para processamento de produtos para saúde em unidades de atenção primária à saúde. Rev SOBECC (Online). [Internet]. 2017 [citado em 01 out 2020]; 22(3):165-70. Disponível em: https://revista.sobecc.org.br/sobecc/ article/view/220. doi:

10.5327/Z14144425201700030008

10. Moreira MN, Silva MPC, Duarte APGM, Resende MP, Amaral JB, Contim D. Educação em saúde no ensino de graduação em enfermagem. Rev Enferm Atenção Saúde [Internet]. 2019 [citado em 01 out 2020]; 8(1):61-70. Disponível em: http://seer.uftm.edu.br/revistaeletro nica/index.php/enfer/article/view/3 362. doi: $10.15253 / 2175$ 6783.2016000500007

RECEBIDO: $25 / 10 / 2020$ APROVADO: 06/07/2021 PUBLICADO: 09/2021 